\title{
Electrochemical Detection of Lactobacillus Rhamnosus in Fermented Food Using Magnetic Immunosensor based on Au- $\mathrm{Fe}_{3} \mathrm{O}_{4}$
}

\author{
Yin Feng*, Yan Liu, Ying Li, Jiamiao Lv, Haiyan Chen \\ School of Life Science, Changchun SCI-TECH University, Changchun 130000, China \\ *E-mail: fengyin1123@sina.com
}

Received: 3 December 2021 / Accepted: 11 January 2022 / Published: 2 February 2022

\begin{abstract}
This study was conducted on the preparation of a high stable and accurate magnetic NPs-based electrochemical immunosensor for the determination of probiotic Lactobacillus rhamnosus (LGG) in fermented foods. A sandwich-like immunocomplex was fabricated using $\mathrm{Au}-\mathrm{Fe}_{3} \mathrm{O}_{4} \mathrm{NPs}$, which were chemically synthesized and conjugated with a specific antibody against the pilus subunit SpaA (anti$\mathrm{SpaA}$ ) and a horseradish peroxidase labeled polyclonal antibody against SpaA (PcAb-HRP), and the resultant immunocomplex was used for modification of the magnetic glassy carbon electrode (PcAbHRP/LGG/anti-SpaA/Au-Fe ${ }_{3} \mathrm{O}_{4}$ NPs/MGCE). The structural analyses using XRD, SEM and FTIR corroborated the successful synthesis of the $\mathrm{Au}-\mathrm{Fe}_{3} \mathrm{O}_{4} \mathrm{NPs}$ and immobilization of biological molecules on the surface of the $\mathrm{Au}-\mathrm{Fe}_{3} \mathrm{O}_{4} \mathrm{NPs}$. Electrochemical studies of the immunosensor using CV, DPV and EIS showed the high sensitivity, accuracy and selectivity of the developed immunosensor to determine LGG. Results revealed that the linear concentration of LGG is from 10 to $10^{9} \mathrm{CFU} / \mathrm{ml}$, and the limit of detection was obtained at $14 \mathrm{CFU} / \mathrm{ml}$. The reproducibility and stability of the immunosensor were investigated, and the results indicated that the acceptable precision, stability, and repeatability of the immunosensor were related to $\mathrm{Au}$ NPs in PcAb-HRP/LGG/anti-SpaA/Au-Fe3O4 NPs which can enhance the signal, electro-catalytic property, biocompatibility and stability of the sensor. The practical capability of the immunosensor for determination of LGG in milk and yogurt was studied and the results illustrated that the components in milk and yogurt matrices did not show any influence on immunosensor detection, implying that the developed immunosensor is a reliable electrochemical LGG sensor in food samples.
\end{abstract}

Keywords: Probiotic Lactobacillus rhamnosus; Magnetic NPs; Sandwich-like immunocomplex; Fermented food; Differential pulse voltammetry

\section{$\underline{\text { FULL TEXT }}$}

(C) 2022 The Authors. Published by ESG (www.electrochemsci.org). This article is an open access article distributed under the terms and conditions of the Creative Commons Attribution license (http://creativecommons.org/licenses/by/4.0/). 